\title{
Electrical characteristics of flash sintering: thermal runaway of Joule heating
}

\author{
R.I. Todd ${ }^{1}$, E. Zapata-Solvas ${ }^{2}$, S. Bonilla, T. Sneddon, P.R. Wilshaw \\ University of Oxford, Department of Materials, Parks Road, Oxford OX1 3PH, UK.
}

${ }^{1}$ Corresponding author: richard.todd@materials.ox.ac.uk

${ }^{2}$ Present address: Instituto de Ciencia de Materiales de Sevilla, Centro mixto CSIC-Universidad de Sevilla, 41092 Sevilla, Spain.

Keywords: flash sintering, YSZ, thermal runaway, electrical conductivity, NTC behaviour

\begin{abstract}
Flash sintering occurs when an electrical field is applied to a heated ceramic powder compact. At a critical combination of field and temperature, a power surge occurs (the "flash event") and sintering takes place in a few seconds. This paper investigates the possibility that this occurs by runaway Joule heating. The resistivity of 3YSZ was measured under the relevant conditions. To a good approximation, resistivity was found to be history-independent and to follow the same relationship with temperature before and after the flash event. These data were used to model the thermal and electrical response of 3YSZ to an applied electric field. All electrical characteristics of the flash event observed experimentally were predicted with a high degree of accuracy. It is concluded that the thermal and electric characteristics of flash sintering are a classical consequence of the negative temperature coefficient of resistivity leading to runaway Joule heating at constant voltage.
\end{abstract}

\section{Introduction}

"Flash sintering" was first reported by Cologna et al in 2010. [1]. In their original report, an initially constant voltage is applied to a ceramic powder compact whilst it is slowly heated in a conventional furnace. At a certain furnace temperature the "flash event" occurs. Over a few seconds the specimen sinters to near full density, as seen by the shrinkage of the specimen. The accompanying electrical response is equally characteristic of the flash event: the current increases, rapidly reaching a pre-programmed limiting value, imposed to limit the power dissipation, after which the applied voltage falls at constant current, resulting in a power spike. The resistance of the specimen falls continuously throughout the flash event. The original report used 3YSZ ( 3 mol\% Y2O3 stabilised zirconia) and direct current but flash sintering has now been demonstrated with a wide range of different ceramics [2-6] and with alternating current [5].

The mechanisms responsible for flash sintering have not yet been established. The main focus of dicussion has been the rapid sintering observed. By extrapolating conventional sintering results, Raj [7] has estimated that a temperature of $\sim 1900{ }^{\circ} \mathrm{C}$ would be required to achieve similar results by heating alone in the short time involved in flash sintering. This is thought to be significantly higher than the temperatures actually attained and several papers have therefore speculated about the possibility of novel matter transport mechanisms associated with the electrical loading of the material $[2,8]$. It has also been suggested that the flash event itself is a consequence of these novel mechanisms, which are considered to involve the nucleation and growth of new defect structures in the crystal [9]. 
The temperature attained by the specimen is highly uncertain owing to the difficulty of making measurements of small specimens under high voltage in short timescales. Raj's estimate of the temperature required to achieve the sintering observed by heating alone is also open to question both because of the very different timescale of flash sintering ( $\sim$ seconds) to the conventional results from which his estimate is extrapolated ( hours) and because of the complex nature of sintering, which involves several competing processes. It is understandable, therefore, that some researchers consider that the rapid sintering is mainly a consequence of the considerable increase in temperature resulting from the Joule heating during the flash event [10] rather than the product of new, as yet unidentified mechanisms.

Investigation of the densification during flash sintering is complicated not only by the multiple mechanisms occurring during the sintering itself but also because it depends on the thermal and possibly electrical aspects of the flash event, which are themselves controversial. Treated as a separate problem, however, these latter aspects are potentially simpler because they are independent of the sintering in the early stages (the current begins to rise before significant densification takes place). Furthermore, there is a classical starting point for the investigation of the electrical response. Most ceramics, whether the conduction mechanism is electronic or ionic in origin, have a negative temperature coefficient (NTC) of resistivity under the relevant conditions. There is therefore the possibility of thermal runaway as Joule heating leads to increased power dissipation (" $V^{2} / R^{\prime}$ ) which leads in turn to a higher heating rate, and so on. This is well known as the problem of self heating of thermistors $[11,12]$ and is consistent with the experimental observation that the resistance of the specimen falls during the flash event.

The aim of this paper is to investigate whether the electrical characteristics of flash sintering of 3YSZ can be explained in terms of thermal runaway. To the authors' knowledge, there are no data in the literature for the variation of resistivity of 3YSZ with temperature under the relevant conditions of high electric field and high temperature. The paper therefore begins by describing preliminary measurements of this relationship. The results are then used to model the electrical and thermal response of 3YSZ to the conditions of flash sintering and finally these predictions are compared with experiment. It extends previous modelling by Grasso et al. [10] by using resistivity data measured under conditions relevant to flash sintering and by considering time-dependent behaviour.

\section{Materials and methods}

2.1 Specimen preparation. All the experiments in this work used bar shaped 3YSZ specimens with a rectangular cross-section. The spacing of the holes for electrical connections and other specimen dimensions were varied slightly to achieve different electric fields but the connections were typically $10 \mathrm{~mm}$ apart and the cross section after sintering was $5.0 \times 1.5 \mathrm{~mm}$ in dimensions. The specimens were made by slip casting of slurries consisting of 55wt\% TOSOH TZ-3Y-E powder and 3wt\% Dispex A40 dispersant in distilled water into plastic moulds on a porous gypsum base. An ultrasonic probe was used to break up any agglomerates in the slurry. Aluminium pins $1.5 \mathrm{~mm}$ in diameter were set in the specimen and removed before samples were completely dried to leave holes for making the electric connections. Final drying was in an oven at $80^{\circ} \mathrm{C}$. The resulting green bodies were approximately $55 \%$ dense. Some of the specimens were fully sintered conventionally in air at $1500^{\circ} \mathrm{C}$ 
before electrical experiments were performed. Sintered densities were measured by the Archimedes method.

2.2 High temperature electrical loading. Experiments in which electric fields were applied to ceramic specimens through a closed electrical circuit were carried out in a modified box furnace. The original furnace door was replaced with an insulating refractory wall which had a quartz window in the middle to allow video recordings to be made of the specimens during flash sintering. The insulating wall also had two small holes for passage of platinum wires from the power supply to the specimen. Inside the furnace the sample was suspended from the two wires by looping them over an alumina frame. The connection between the wires and the specimen was made by inserting the wires through the holes in the specimens without the aid of conductive paint. A commercial DC power source (360 V, 15 A, 1500 W, Elektro-Automatik, Viersen, Germany) power supply was used and data was logged using Labview software.

The basic flash sintering experiments were similar to those of Cologna et al. [1]. Green samples were initially heated in the furnace at a rate of $7.5^{\circ} \mathrm{C} / \mathrm{min}$ to $600^{\circ} \mathrm{C}$ and $5{ }^{\circ} \mathrm{C} / \mathrm{min}$ at higher temperatures. Electric fields of between 80 and $1200 \mathrm{~V} / \mathrm{cm}$ were applied. The power supply was programmed to apply a constant voltage but was usually subject to a maximum current (typically $0.5 \mathrm{~A}$ ). At a certain furnace temperature the flash event occurred as described in the Introduction. The electrical and sintering responses and the conditions of furnace temperature and applied electric field under which the flash event occurred were very similar to those reported in reference [1] and other papers. Examples are in Figs. 5 and 6 below.

Similar experiments were conducted on dense, pre-sintered specimens but under current control from the start. These are described in more detail in section 3.1.

The relatively small change in dimensions caused by sintering was ignored in calculating the electric field and resistivity.

2.3 Estimation of furnace and specimen temperatures. All furnace temperatures used in this work are the effective temperature of the furnace atmosphere at the specimen position. These were obtained by an initial calibration of the temperature difference between a thermocouple held at the specimen location and the furnace control thermocouple over the range of furnace settings used.

In the conditions of interest for flash sintering, the passage of electrical current through the specimen always leads to sufficient Joule heating to raise the specimen temperature significantly above the furnace temperature, even before the flash event itself. Accurate direct measurement of the specimen temperature using thermocouples is not compatible with the electrical loading of the specimen. Pyrometry of small specimens with uncertain emissivity is not accurate and can only measure the surface temperature, which may be much lower than the temperature inside the specimen, as will be shown below. In this work, therefore, we follow Raj [7] in estimating the specimen temperature by assuming there are no temperature gradients in the specimen and equating the electrical power dissipation to the radiative heat loss using eq. 1 : 
in which $T$ is the specimen temperature, $T_{0}$ is the temperature of the furnace atmosphere, $W$ is the power dissipation, $A$ is the surface area of the specimen, $\varepsilon$ is the emissivity and $\sigma$ is Stefan's constant. Although this method suffers from some of the same disadvantages as were described for pyrometry, it does give an average over the specimen length rather than sampling a single point and is easier to execute.

The choice of emissivity is particularly problematic as it can be expected to vary with density during sintering as well as with surface roughness, wavelength and temperature. Measurements of the total hemispherical emissivity, in which all wavelengths and directions are included are most appropriate to the present application. Most data for YSZ are for plasma or flame sprayed samples and typical values are $0.3^{\sim} 0.6$ in the temperature range of interest $[13,14]$. However, different microstructures can lead to values of 0.9 or greater in oxide ceramics [15]. A constant intermediate value of 0.7 was chosen for this work, which is consistent with the measurements of Tanaka et al. [16] on sintered zirconia.

\section{Electrical properties of $3 Y S Z$ under conditions relevant to flash sintering}

3.1 Preliminary investigation of time dependence. In order to model flash sintering, at least an empirical description of the voltage-current-temperature response of the ceramic under the relevant conditions is required. We begin with a preliminary investigation of the time dependence of resistivity. Figure 1 shows the results of experiments on a fully dense, presintered specimen in which the current was set to different values for periods of several tens of seconds at a constant furnace temperature of $970{ }^{\circ} \mathrm{C}$. The schedule stepped the current up to a maxium value of $0.95 \mathrm{~A}$ and stepped down through the same values.

Several interesting features are evident from this plot. The most important for the modelling is that the time and history dependence of the current-voltage relationship was small once the initial transient at each change of current was completed. Close examination shows that there is a tendency for the voltage to continue to diminish after the initial transient during the first few steps and it is evident that the second application of each current requires a slightly smaller voltage than the first. However, these effects are small compared with the effect of temperature described in the next section and occur on a longer timescale and with higher currents than those leading up to the flash event.

Two other features are worthy of note. The first is that on the initial application of current, there is a sharp voltage spike which corresponds to a "flash event" as the power supply forces current through the specimen. Each subsequent change in current also has a small voltage transient at the start (see inset on Fig. 1). It may be argued that the materials properties change during the rapid initial transient but it will be shown in the next section that any such change is also small. The models to be presented will also show that such flash events can be explained in terms of Joule heating. It is therefore reasonable to model the electrical response of these materials as being time independent. 
It is also evident that the specimen apparently exhibits negative differential resistance, in which the voltage required to maintain a current is lower for higher currents. The models presented later in this paper show this to be a consequence of the NTC characteristic of the resistivity, in which the reduction in resistivity caused by the extra Joule heating on increasing the current more than compensates for the increased electromotive force required to increase the current when the resistivity is constant. A consequence of this behaviour is that the current is insensitive to the applied voltage. This and the negative differential resistance lead to the instabilities in the voltage applied by the power supply as it attempts to maintain the higher current values (Fig. 1).

3.2 Resistivity-temperature relationship. We proceed assuming time-independent Ohmic behaviour and an inverse Arrhenius temperature dependence of the resistivity:

$\rho=\rho_{0} \exp (Q / R T)$

where $Q$ is the activation energy for the conduction mechanism and $R$ the gas constant. This relationship is often obeyed by ionic and semi-conducting ceramics.

Figure 2 shows several sets of results plotted as $\ln \rho$ against $1 / T$. The results included are for (a) green bodies in constant voltage experiments well before the flash event, (b) the same specimens when a steady state has been established after the flash event, and (c) specimens conventionally presintered to full density and then tested with a range of furnace temperatures and imposed currents, as in Fig. 1 but with only the minimum amount of time required to achieve a steady state allowed, in order to minimise time-dependent effects.

The straight line shown is the line of best fit to the results from green bodies well before the flash event and corresponds to an activation energy $Q$ of $171 \mathrm{~kJ} \mathrm{~mol}^{-1}$ and a pre-exponential $\rho_{0}$ of $1.08 \times$ $10^{-6} \Omega \mathrm{m}$. These values were used with eq. 2 for all the modelling in this paper (i) because the temperature adjustment required using eq. 1 is a minimum for these pre-flash specimens, thus minimising the effect of temperature gradients and the uncertainty in emissivity, and (ii) because these results are most relevant to the conditions before and during the flash event on which the modelling is focused.

However, it is evident in Fig. 2 that the line of best fit for resistivity before the flash event extrapolates reasonably well to the post-flash results from the same specimens and to those from pre-sintered specimens under constant current, implying a single underlying conduction mechanism that is approximately independent of time and specimen history, as suggested in the previous section. Most importantly, the conformity of the specimens to the same resistivity-temperature relationship before and after the flash event shows that there is no significant change in specimen properties either during the period under voltage before the flash or during the flash event itself.

The pre-flash specimens are more porous than the other specimens in Fig. 2. This must have an effect on the resistivity and indeed results not reported in detail here showed that presintering the green specimens to increase their initial density reduced the applied field required to activate the flash event for a given furnace temperature. The straight line fit in Fig. 2 is therefore strictly only applicable to initially unsintered specimens. The reasonable extrapolation to fully sintered 
specimens despite this is thought to be because the effect of porosity is small compared with the dominating effect of temperature: the range of experimental resistivities in Fig. 2 covers 3.5 orders of magnitude, but the effect of porosity in the present case is expected to be less than one order of magnitude [17], which is comparable with the scatter in the plot. Similar comments apply to the small time and history dependence described in section 3.1. It is therefore reasonable to use the straight line fit to the pre-flash results to model the whole flash event.

In summary, although relative density and history have some influence on electrical properties of 3YSZ, both effects are overwhelmed by the very large resistivity changes occurring because of the temperature change of the specimen. The modelling is therefore justified in assuming the electrical properties are controlled by the time-independent resistivity embodied in eq. 2 .

\section{Other physical properties used in the modelling}

The competition between surface heat loss and Joule heating is an important aspect of flash sintering. This is the basis of the modelling and the estimation of the specimen temperature via eq. 1. A value of 0.7 was used for the emissivity as described in section 2.3. For completeness, convective heat loss was also included in the dynamic, non-uniform (DNU) model of section 6 . The heat transfer coefficient of free air has typical values between 5 and $25 \mathrm{~W} \mathrm{~m}^{-2} \mathrm{~K}^{-1}$ [18]. A mid-range value of $10 \mathrm{Wm}^{-2} \mathrm{~K}^{-1}$ was used in the model. The modelling showed that radiative heat loss is always dominant in the conditions of interest, convection affecting the specimen temperature by typically only a few $\mathrm{K}$.

The thermal conductivity is expected to be affected by the relative density and temperature of the specimen but this is ignored in the model and a constant value of $2.7 \mathrm{~W} \mathrm{~m}^{-1} \mathrm{~K}^{-1}$ is used [19]. Similar comments apply to the density and specimen radius. All physical values used in the model are summarised in Table 1.

\section{Initial Considerations - Static Model with Uniform Specimen Temperature (SU Model)}

Raj [7] has used the assumption of a uniform specimen temperature to relate electrical power to specimen temperature, $T$, under steady state conditions. The same assumption of uniform temperature is instructive in understanding the conditions under which thermal runaway can occur at constant applied field. A long cylindrical specimen of radius $r_{0}$ with an applied electric field $E$ is considered (Fig. 3a). The power per unit length of the cylinder dissipated in Joule heating, $W_{+}$, is

$W_{+}=\frac{E^{2} \pi r_{0}^{2}}{\rho_{0}} \exp \left(-\frac{Q}{R\left(T_{0}+\Delta T\right)}\right)$

where $T_{0}$ is the furnace temperature and $\Delta T=T-T_{0}$ is the amount by the specimen temperature exceeds $T_{0}$.

The rate of radiative heat loss per unit length, $W_{-}$, is given by:

$W_{-}=2 \pi r_{0} \sigma \varepsilon\left(\left(T_{0}+\Delta T\right)^{4}-T_{0}^{4}\right)$

where $\sigma$ is Stefan's constant and $\varepsilon$ is the emissivity. 
Figure $4(\mathrm{a})$-(c) shows plots of $W_{+}$and $W_{-}$against $\Delta T$ for a constant field of $100 \mathrm{~V} / \mathrm{cm}$ and three different furnace temperatures $T_{0}$. A value of $1.5 \mathrm{~mm}$ has been chosen for $r_{0}$ to give similar cross sectional area to the specimens used in our experiments. The power dissipated in Joule heating $W_{+}$ must always be greater than the cooling contribution from radiation $W_{-}$for sufficiently small values of $\Delta T$. However, when the furnace temperature is relatively low (e.g. $T_{0}=1250 \mathrm{~K}$, Fig. 4a) $W_{-}$ becomes equal to $W_{+}$with only a small excess temperature $(\Delta T=20 \mathrm{~K})$ and the specimen temperature becomes stable at $T_{0}+\Delta T=1270 \mathrm{~K}$. As $T_{0}$ increases, $\Delta T$ also increases so that when $T_{0}=$ $1320 \mathrm{~K}$ (Fig. 4b), $\Delta T=59 \mathrm{~K}$ and the stable specimen temperature is $1379 \mathrm{~K}$. The specimen temperature remains stable until $T_{0}$ increases to the critical temperature of $1339 \mathrm{~K}$ (Fig. 4c) at which point $\Delta T=117 \mathrm{~K}$, the specimen temperature is $1456 \mathrm{~K}$ and the heating and cooling curves are tangential to one another. As $T_{0}$ increases beyond this point, the power input always exceeds the radiative heat loss and the specimen temperature becomes unstable, increasing without limit and at an accelerating rate because of the ever increasing gap between the heating and cooling curves. The present paper argues that this is the essence of the flash event. It originates in the exponential reduction in resistivity and consequent increase in electrical power dissipation at constant applied voltage $\left(V^{2} / R\right)$ as the specimen temperature increases.

The above discussion is in the context of an experiment in which the applied field is held constant and the furnace temperature is gradually increased. Similar arguments show that in experiments with constant furnace temperature there is a critical applied electric field above which the same thermal runaway occurs.

Figure $4 d(a)$ shows that the critical condition for the flash event to take place is when $W_{+}=W$ - and $\frac{+}{d \Delta T}=\frac{d \Delta T}{d \Delta T}$. The critical electric field $E_{\text {crit }}$ and critical excess temperature $\Delta T_{\text {crit }}$ for a given furnace



$\stackrel{\text { and for }}{\Delta T_{\text {crit }} \approx \frac{\Delta T_{\text {criRt }} T_{0}}{Q-5 R T_{0}}}$

Equations 5 and 6 enable the quantitative prediction of the relationship between $E_{\text {crit }}$ and $T_{0}$. These are compared with experiment in Fig. 5. The agreement is good considering the scatter in the resistivity data and the uncertainties involved (e.g. the emissivity value). The approximation in eq. 6 leads to errors in $E_{\text {crit }}$ of less than $4 \%$ compared with the exact solution over the temperature range of Fig. 5.

Equations 5 and 6 also predict that for a given furnace temperature, $E_{\text {crit }}$ decreases with reducing $\rho_{0}$, such as might occur when using pre-sintered specimens, as was noted in section 3.2 to be the case in practice, and with larger cross-sectional dimension, $r_{0}$. The latter prediction remains to be tested.

The thermal runaway at constant field is accompanied by an increase in current and in practice the power supply switches to current control at a pre-determined limiting current to prevent its power capacity from being exceeded. This situation is shown for the example of $E=100 \mathrm{~V} / \mathrm{cm}, T_{0}=1339 \mathrm{~K}$ 
and a limiting current of $0.5 \mathrm{~A}$ in Fig. 4(d). The switch to current control occurs when $\Delta T=407 \mathrm{~K}$ and corresponds to the peak in power dissipation. Although the power dissipated then reduces, it remains above the radiative heat loss until $\Delta T=488 \mathrm{~K}$ at which point the specimen temperature stabilises at $1827 \mathrm{~K}\left(1554^{\circ} \mathrm{C}\right)$. The falling resistivity with increasing temperature ensures that stability is attained under constant current owing to the " ${ }^{2} R$ " electrical power dissipation.

This SU model has given useful insight to how thermal runaway can lead to the flash event, but it cannot predict its dynamics and is not sufficiently accurate for situations where high thermal gradients, and therefore current gradients are expected. A numerical model is developed in the next section to provide such predictions.

\section{Dynamic model with non-uniform temperature (DNU Model)}

6.1 Description of the model. The non-uniform, dynamic model again assumes a long cylindrical specimen. The current flows along the length of the specimen under the action of an electric field. The cross section of the specimen is divided into concentric cylindrical elements much smaller in thickness than the specimen (Fig. $3 \mathrm{~b}$ ). It is assumed that the temperature and current are constant over the radial thickness of each element. The elements have no physical interpretation but must be thin enough for this assumption to hold. 20 cylindrical elements were used in the results presented here; increasing the number of elements to 90 made no appreciable difference to typical predictions. An outer radius $r_{0}=1.5 \mathrm{~mm}$ was used in the present paper, as for the SU model, again to give similar dimensions to the experimental specimens.

The model operates at constant furnace temperature and loops around increments of time with either constant field or constant current. The specimen temperature is initially set to the furnace temperature. In each step, the resistance $R$ of each element $j$ is calculated from its dimensions and its resistivity, which is determined from the instantaneous temperature of the element. The net heating rate of the element is calculated from the sum of the electrical power input $E^{2} / R$ per unit length and the thermal energy conducted across the inner and outer boundary of element $j$ with the neighbouring elements $j+1$ and $j-1$ along with the heat capacity and density. The temperature gradients are taken as $\left(T_{j \pm 1^{-}} T_{j}\right) / t$ where $T$ denotes temperature and $t$ is the element thickness. The central element has only one boundary and the outermost element loses heat from its outer boundary by radiation according to Stefan's law (incorporating the emissivity) and by convection. The total current in the specimen is determined by adding the contributions from each element.

A time increment is then chosen to give a maximum temperature change of $0.01 \mathrm{~K}$ in any element and the temperatures of the elements are updated. When operating in constant current mode, the electric field is also updated to bring the total current back to the specified value via Ohm's law. The next time increment then begins.

The above scheme was implemented in Fortran 77.

6.2 Comparison of DNU model with experiment (1): electric field versus flash temperature. We first compare the DNU model's prediction of the of the $E_{c r i t}$ and $T_{0}$ relationship with experiment. For each furnace temperature $T_{0}$ the model was run for applied fields spaced by $50 \mathrm{~V} / \mathrm{cm}$. The mean of the highest field at which no flash occurred and the lowest field at which a flash event occurred within $300 \mathrm{~s}$ of the field being applied are marked on Fig. 5. It is evident that the prediction is very similar 
to that of the SU model and so is also in good agreement with the experimental results. The good agreement with the SU model in this case is because, as will be seen in Section 6.4, the DNU model shows that thermal gradients in the specimen are small before the flash event, as assumed by the SU model.

6.3 Comparison of DNU model with experiment (2): full electrical response with time. Figure 6 compares the predicted and experimental results of current, electric field, power and specimen resistance against time relative to the flash point for a typical example of an initial field of $100 \mathrm{~V} / \mathrm{cm}$ and a current limit of $0.55 \mathrm{~A}$. Both were initially close to the critical condition of Fig. 4 (c), but the experiment was conducted under slow heating whereas the DNU model was run isothermally with furnace temperature $T_{0}=1341 \mathrm{~K}$ (just above the critical temperature in the SU model of $1339 \mathrm{~K}$ ). To allow comparison, the time was arbitrarily defined to be zero in both cases when the switch from voltage to current control occurred.

It is evident from Fig. 6 that the model quantitatively predicts all aspects of the electrical behaviour around the flash event with considerable accuracy.

\subsection{Comparison of DNU model with experiment (3): constant field, initial lead time ("incubation} time") and localisation of heating. Having established the success of the model in predicting the main electrical aspects of the flash event, we now use it to examine some details of the phenomenon using an applied field of $300 \mathrm{~V} / \mathrm{cm}$ as an example, intially without a current limit. In order to activate the flash event, a constant furnace temperature of $1115 \mathrm{~K}$ was modelled, which is 5 $\mathrm{K}$ higher than the ideal flash temperature of $1110 \mathrm{~K}$ estimated for these conditions using the SU model. Figure 7 shows the predicted total current against time after the application of the electric field. The current (and therefore the power dissipated at constant voltage) remains at a low level $(<0.1 \mathrm{~A})$ for almost $178 \mathrm{~s}$ and then, within a few seconds, suddenly increases to very high levels. The current against time for a constant voltage experiment with the same applied field is also shown in Fig. 7. For the purpose of comparison, the time for the experiment is arbitrarily fixed to the model at the point when the current is $0.5 \mathrm{~A}$. It is evident that the quantitative agreement between the model and the experimental result is again excellent.

Given the conflicting interpretation attached to it by some authors [9], it is worth exploring the lead time before the flash event in more detail. Figure 8 (a) shows the predicted temperature distribution in the specimen for times up to $175 \mathrm{~s}$ after the application of the electric field. Although the current is low, the temperature of the specimen rises continuously during most of this initial period and remains reasonably constant across the specimen cross section because the thermal conductivity is sufficient to homogenise the temperature. Significant specimen heating considerably before the onset of the flash event was also apparent in the experimental videos. The rate of heating is slow, however, both because the specific power of Joule heating is low compared with the volumetric heat capacity when the specimen is at the relatively low specimen temperatures before the flash event and because it is shadowed closely by the rate of radiative heat loss for a wide range of excess temperature, $\Delta T$, as in Fig.4c.

Eventually, the exponential rise of Joule heating with temperature dominates the restraining effects of heat capacity and radiative heat loss, and rapid heating occurs. The model shows that an 
additional factor is that thermal conductivity is no longer sufficient to homogenise the temperature with such rapid heating, leading to severe current and temperature localisation. Figure 8 (b) shows that the specimen temperature rises several hundred $\mathrm{K}$ between 175.00 and $177.80 \mathrm{~s}$ and thereafter the specimen becomes significantly hotter in the centre than at the edge. At $178.00 \mathrm{~s}$ the temperature at the centre of the specimen reaches $2155 \mathrm{~K}$ and after only a further $0.02 \mathrm{~s}$ is predicted to be $4820 \mathrm{~K}$, higher than the melting point of zirconia. At this time, the current density at the centre of the specimen is more than 3 orders of magnitude greater than that at the edge (Fig. $8(\mathrm{c})$ ). Even so, the predicted rate of temperature increase at the specimen edge is several thousand $\mathrm{K} \mathrm{s}^{-1}$. The SU model demonstrates that this localisation is not necessary for runaway heating to occur but it can bring forward its onset significantly if the heat generated by Joule heating cannot escape from the specimen interior. The thermal diffusivity and specimen size are therefore important in determining the precise conditions for the flash event. (The specimen size also affects flash sintering because of the different scaling of $W_{+}$and $W_{-}$with cross sectional dimensions as shown in eq. 5. )

Although the increase in temperature in Fig. 8 (b) is extreme, the total currents predicted are modest (e.g. $3.7 \mathrm{~A}$ at $178.00 \mathrm{~s}$ ) so these conditions can easily be approached experimentally. The specimen shown in Fig. 8(d) was run with the same applied field of $300 \mathrm{~V} / \mathrm{cm}$ and the current limit of the power supply was $2 \mathrm{~A}$. This was sufficient to allow the observation of the predicted localisation as a stripe of well sintered (or perhaps partially melted) material between the two electrodes which is detached from the partially sintered surrounding material. The geometry of the specimen is different from the cylindrical assumption of the model but the cross sectional dimensions are similar and there can be little doubt that it is a consequence of the same instability as is predicted by the DNU model (Fig. 8b).

6.5 Current limit - stabilisation of Joule heating and its limitations. The previous section shows that if a sufficiently high electric field is applied without a limit to the current, catastrophic runaway heating and localisation occur which prevent uniform sintering. As shown for the SU model in Fig. 4(d), the imposition of a current limit is expected to stabilise the specimen temperature and the DNU model confirms this. Figures 8 (b) and (c) include the predicted temperature and current density profiles well after the switch to constant current for the starting conditions of the previous section but with a $0.5 \mathrm{~A}$ current limit. The temperature stabilises at a level slightly higher than the temperature at $177.8 \mathrm{~s}$ at which the current reached this limit. The constant current also reduces the extent of localisation although with temperatures at the centre and edge of the specimen of 1872 and $1768 \mathrm{~K}$ respectively, a considerable temperature gradient remains. For specimens with thicker cross sections, very considerable differences in temperature between the centre and the outside surfaces are predicted, even under current control. A lower temperature at the specimen edge was apparent in the experimental videos.

We examine the temperature distribution around the flash event for a wider range of conditions by comparing the model's predictions for specimens with initial electric fields of 100 and $1000 \mathrm{~V} / \mathrm{cm}$ and furnace temperatures of 1341 and $950 \mathrm{~K}$ respectively and switching to current control at $0.5 \mathrm{~A}$. These temperatures were chosen to be a few $\mathrm{K}$ above the respective flash temperatures. Fig. 9 (a) shows the predicted temperatures at the centres and edges of the specimens. Substantial excess heating $\Delta T$ above the furnace temperature before the flash event is predicted in both cases but, using the temperatures $10 \mathrm{~s}$ before the switch to current control for comparison, is greater for the 
low $E /$ high $T_{0}$ specimen ( $190 \mathrm{~K}$ ) than for the high $E /$ low $T_{0}$ specimen ( $120 \mathrm{~K}$ ), in qualitative accord with eq. 6 . The rate of temperature increase at the flash event is much greater for the high $E /$ low $T_{0}$ case and the difference in temperature between the centre and the edge shows a sharp maximum of $\sim 300 \mathrm{~K}$ at the flash point (Fig. 9b) owing to the onset of localisation before the current limit is reached. After the switch to current control, the specimen temperatures become similar but are a few tens of degrees lower and with a bigger gradient from centre to edge in the low $T_{0}$ specimen because of greater heat loss to the cooler furnace atmosphere.

The prevelance of temperature gradients during flash sintering, even under constant current conditions, shows that pyrometer measurements of the surface temperature and estimates of specimen temperature using eq. 1 can significantly underestimate the interior temperature of the specimen.

\section{Discussion}

7.1 Runaway Joule heating as the origin of the flash event. The DNU model contains only physically meaningful parameters which are directly measured in this work or are physical constants available from the literature. The activation energy and pre-exponential for resistivity are fitted to results obtained before the flash event. The model results in Figs. 5-8 are therefore genuine predictions of the flash event with no parameters adjusted to fit the event itself (except for the zero point of time where required because of the different conditions for model and experiment). The effects predicted with good quantitative agreement with experiment include the slow initial period of heating (called the "incubation time" by Naik et al. [9] and referred to as the "lead time" by the present authors), the relationship between the applied field and the flash sintering temperature and the detailed evolution of applied field, current, resistivity and power during the flash event itself, including the prediction of localisation of heating under conditions of constant electrical field and high current limit.

This excellent and wide ranging agreement with experiment is convincing evidence that the electrical and thermal response of 3 YSZ during flash sintering is primarily a thermal runaway effect resulting from the dependence of Joule heating on local specimen temperature through its effect on the resistivity.

7.2 Comparison with evidence in the literature. Acceptance of Joule heating as the primary origin of flash sintering enables other aspects of the phenomenon which have been reported to be understood. For instance, the finding of Francis et al. [20] that green compacts with larger particle size need a higher temperature to induce the flash event at constant voltage can be explained in terms of eqs. 5 and 6 (larger particle size means fewer particle-particle contacts and therefore higher resistivity through $\rho_{0}$ ). This is similar to the explanation for the reduction in $E_{\text {crit }}$ with partial presintering mentioned in section 5 .

Cologna et al. [21] noted two conditions for the onset of the flash event. One of these, that the furnace temperature needs to exceed a critical value for the flash event to occur, has already been discussed in terms of the need to exceed the critical point of Fig. 4 (c). The other is that the specific 
power dissipation needs to exceed a level of about $10 \mathrm{~mW} \mathrm{~mm}^{-3}$ at the onset of the flash effect. The DNU model correctly predicts this as is shown in Fig. 10, which is an Arrhenius plot for specific power dissipation. The form of this predicted plot closely resembles experimental plots in references [1] and [21]. Ceramics have typical volumetric heat capacities in the range of 2-4 $\mathrm{mJ} \mathrm{mm}^{-3} \mathrm{~K}^{-1}$. A power of $10 \mathrm{~mW} \mathrm{~mm}^{-3}$ therefore corresponds to a Joule heating rate of several $\mathrm{K}$ per second if cooling is ignored. When the power is less than this, heating is slow and can be reduced by radiative cooling (Fig. 4 (a) and (b)). When the specific power dissipation is greater than this, then the rate of temperature rise is rapid leading to thermal runaway. Localisation is also important in determining the critical heating rate. The characteristic distance for heat conduction is $\sim \sqrt{\alpha t}$ where $\alpha$ is the thermal diffusivity and $t$ is the time. With the values in Table 1 it can be deduced that heat travels about $1 \mathrm{~mm}$ in $1 \mathrm{~s}$ in 3YSZ. If the heating is significant on the timescale of a few seconds with the present specimen dimensions, heat cannot reach the surface of the specimen by conduction and localised runaway occurs. The same consideration also shows that localised heating of grain boundaries, which has been suggested to play a part in flash sintering [1], cannot be sustained for more than $\sim 1 \mu$ s before thermal conduction homogenises the temperature and therefore does not provide a tenable explanation for sintering on the observed timescale of several seconds.

The concept of Joule heating as the origin of the flash effect also explains its apparent generality: the phenomenon has now been reported in many different ceramics, including those normally considered ionic conductors such as YSZ, electronic conductors and semiconductors [2-6]. Although the physical mechanisms of conduction vary amongst these materials, they have in common the decreasing electrical resistivity with temperature which the present work suggests to be the origin of the flash event. Flash sintering of $\mathrm{BaTiO}_{3}$, which is well known to exhibit positive temperature coefficient (PTC) of resisitivity behaviour, has also been reported recently [22]. However, it should be noted that this behaviour is seen over a narrow temperature range around the Curie temperature, typically $100-200{ }^{\circ} \mathrm{C}$ depending on grain size, stoichiometry and doping. At the higher temperatures relevant to flash sintering, NTC behaviour is exhibited [23].

Finally, the high specimen temperatures predicted (typically $1500-1600{ }^{\circ} \mathrm{C}$ ) are the most obvious starting point for explaining the emission of light by the specimens during the flash event [24].

7.3 Explanation of the lead time ("incubation time"). It is particularly important to discuss the long lead time before the flash event in constant voltage experiments because it has been interpreted as being an "incubation time" indicative of a process involving the nucleation and growth of nuclei of defect structures which eventually produce the flash event in an avalanche. In particular, Naik et al. [9] have suggested that the Joule heating around the flash event is a consequence of this avalanche rather than the cause of it. The present work does not support this view and offers a convincing alternative explanation for both the flash effect and for the long lead time before it. The most direct argument against the proposed nucleation of a new regime of conductivity is the data in Fig. 2 showing that the difference in the resistivity-temperature behaviour of the material before and after the flash event is relatively small. However, the a priori quantitative prediction of the flash event based only on data measured before it occurs and the classical NTC resistivity which forms the basis of the model via eq. 2 is a convincing demonstration that the data of Fig. 2 offer a complete explanation of the thermal and electrical characteristics of flash sintering without needing contributions from extra, time dependent mechanisms. 
Naik et al. [9] also draw attention to the extreme non-linearity of the relationship between the lead time and the applied field in isothermal tests. This has a ready explanation in terms of Joule heating, as is evident in Fig. 4. When the applied field at constant furnace temperature (or as presented in Fig. 4, the furnace temperature at constant applied field) is below the critical tangency condition of Fig. 4 (c), the flash event will not take place no matter how long the experiment is left to run. However, in increasing the field or temperature from just below this critical value to just above it, the lead time changes from being infinite to finite and shortens rapidly as the heating and cooling curves become more widely separated. This is demonstrated using the DNU model in Fig. 11, which shows a semi-logarithmic plot of applied field against predicted lead time for different furnace temperatures in isothermal tests. These results cannot be compared directly with those of Naik et al. because of the different materials involved but the increase in incubation time from a few seconds to very large values in reducing the applied field by only a few $\mathrm{V} / \mathrm{cm}$ reported by Naik et al. is evident nevertheless. Although lead times of arbitrarily long duration can be explained in this way, the extreme sensitivity of lead times to the applied field for durations of over $1000 \mathrm{~s}$ in Fig. 11 suggests that the observation of lead times $\sim 10^{4} \mathrm{~s}$ by Naik et all. [9] is also a product of second order effects occurring on this timescale. One obvious possibility is the slow initial sintering of the powder compact. This would gradually reduce the resistivity of the compact, slowly increasing the heating at constant voltage and eventually precipitating the flash event. The small time-dependence of resitivity noted in Fig. 1 would have a similar effect.

7.4. The resistivity-temperature relationship. The excellent quantitative agreement between the modelling and experiment in the present paper relies on the resistivity-temperature relationship of eq. 2, which was fitted to the data taken before the flash event. Notwithstanding their success in predicting the main features of the flash event, it is important to recognise their limitations. Sources of inaccuracy include the lack of accounting for the effect of sintering and the relatively small time dependence, as discussed in section 3. The estimation of specimen temperature is also approximate because of the uncertainty in emissivity and the assumption of uniform temperature in eq. 1, which the model shows to be inappropriate under some conditions. Further considerations are the imperfect geometry of the specimens and the fact that the measurements include the contact resistance of the specimen with the electrodes; this is fine for estimating the global electrical response of the system but further reinforces the fact that the electrical property data in this work are essentially empirical and include several distinct physical effects. The model is thought to predict the main features of the flash event successfully because the effect of temperature on the resistivity is sufficiently large to over-ride these secondary effects in most circumstances. However, there are certainly details of the phenomenon which require a more comprehensive, physically based description of the material behaviour.

3YSZ is well known as an ionic conductor. As has been pointed out by Muccillo et al. [25], however, it is not likely that the present conditions of high current can be achieved by ionic conduction alone owing to the need to supply large amounts of oxygen at the cathode for ionic conductivity to be sustained. Indeed, the apparent activation energy of $171 \mathrm{~kJ} \mathrm{~mol}^{-1}$ in this work is much higher than that for ionic conduction, which is $72 \mathrm{~kJ} \mathrm{~mol}^{-1}$ [26]. The actual mechanism of conduction must be electronic and the evidence in this work is that the mechanism is time-independent, at least on the 
timescale of the flash event, and is the same before and after the flash event has taken place. It does not involve the incubation of a distinct defect structure causing a change in conduction mechanism and is probably not strongly connected with the reduction of the specimen, which also leads to an electronic contribution to conductivity [25] but is relatively slow and is a function of total charge passed. Instead, it seems that the results of Fig. 2 represent a more innate semiconducting behaviour with high activation energy and which therefore dominates at high temperature and possibly high electric field. Electrical data on ceramics under the conditions of flash sintering are scarce and further progress will require a more detailed investigation of the thermo-electrical response, including the use of four point resistance measurements to separate the material behaviour from the contact resistance.

7.5 Densification behaviour. Although this work adds significantly to the understanding of the electrical and thermal features of flash sintering, it should be emphasised that it gives little direct information about the rapid densification of the specimen. Under many of the experimental conditions used here, the specimens sintered to full density and developed uniform grain sizes along the length and across the section. The videos showed that this took place in a few seconds around the flash event, as in the original report of the phenomenon [1]. The modelling suggests that the centre of our specimens typically reached $\sim 1600{ }^{\circ} \mathrm{C}$ and the outer surface $\sim 1500{ }^{\circ} \mathrm{C}$ (e.g. Fig. 9a). The temperature estimate depends on the value of emissivity assumed but is in broad agreement with the numerical estimates of Grasso et al. [10]. Changing the emissivity from 0.7 to 0.5 or 0.9 makes a typical difference of only a few tens of Kelvin to the predicted temperatures in the constant current phase after the flash event. The experiments of Baraki et al. [27], based on thermal expansion measurements indicate temperatures similar to those of the predictions. An increase in temperature to almost $1700^{\circ} \mathrm{C}$ is also the most obvious explanation of the lattice expansion during flash sintering of 8YSZ measured using synchrotron radiation diffraction by Akdogan et al. [28], although these authors do not interpret their results in this way.

Though below Raj's estimate [7] of the temperature required for sintering on the observed timescale $\left(1900^{\circ} \mathrm{C}\right)$, the temperatures evidently attained during flash sintering are well above the temperatures used for conventional sintering of the present powders $\left(\sim 1350^{\circ} \mathrm{C}\right)$. In view of the considerable inaccuracy in extrapolating results from conventional sintering to the timescales of flash sintering, whether or not rapid heating to 1500 or $1600{ }^{\circ} \mathrm{C}$ is sufficient to cause the observed densification without the requirement for new transport mechanisms related to the electric field remains an open question.

Although the mechanistic details of densification remain uncertain, the DNU model does provide useful indications of the practical conditions required for successful flash sintering. The use of high fields in flash sintering enables a lower pre-heat temperature to be used but leads to a rapid and non-uniform increase in temperature which is likely to crack the specimen (Fig. 9). The final specimen temperature achieved, which can be expected to determine the extent of sintering, is controlled primarily by the limiting current. However, too high a limiting current gives more scope for temperature gradients and catastrophic localisation of the current and heating in the preceding constant field stage. For practical application of flash sintering, therefore, more sophisticated electrical control profiles which allow high currents to be achieved without localisation may be desirable. 


\section{Summary}

It is shown that the main electrical and thermal characteristics of flash sintering of 3YSZ can be predicted accurately through the observed inverse Arrhenius dependence of resistivity on temperature. The modelling uses independently measured physical properties and makes absolute predictions with no adjustable. The effects correctly predicted include the slow initial period of heating (lead time), the influence of applied field on the flash sintering temperature, the localisation of heating under constant field, the influence of powder particle size and the detailed quantitative evolution of applied field, current, resistivity and power during the flash event itself. The electrical behaviour of the ceramic was found to be similar before and after the flash event and the time dependence of resistivity was relatively small. The flash event is thus seen to be a consequence of runaway Joule heating under voltage control caused by the rapid reduction of resistivity with increasing temperature.

\section{Acknowledgements}

The authors are grateful for the funding of part of the experimental work in this paper by Dstl, UK under CDE Contract Number DSTLX1000065524.

\section{References}

1. Cologna M, Rashkova B, Raj R. Flash sintering of nanograin zirconia in $<5 \mathrm{~s}$ at 850 으. J Am Ceram Soc 2010;93:3556-9.

2. Cologna M, Francis SCJ, Raj R. Field assisted and flash sintering of alumina and its relationship to conductivity and MgO-doping. J Eur Ceram Soc 2011;31:2827-37.

3. Karakuscu A, Cologna M, Yarotski D, Won J, Raj R, Uberuaga BP. Defect structure of flash sintered strontium titanate. J Am Ceram Soc 2012;95:2531-6.

4. Prette ALG, Cologna $\mathrm{M}$, Sglavo V, Raj R. Flash-sintering of $\mathrm{Co}_{2} \mathrm{MnO}_{4}$ spinel for solid oxide fuel cell applications. J Pow Sour 2011;196:2061-5.

5. Muccillo R, Muccillo ENS, Kleitz M. Densification and enhancement of the grain boundary conductivity of gadolinium-doped barium cerate by ultra fast flash grain welding. J Eur Ceram Soc 2012;32: 2311-16.

6. Zapata-Solvas E, Bonilla S, Wilshaw PR, Todd RI, Preliminary investigation of flash sintering of SiC, J Eur Ceram Soc 2013;33: 2811-2816.

7. Raj R. Joule heating during flash-sintering. J Eur Ceram Soc 2012;32:2293-2301.

8. Raj R, Cologna M, Francis JSC. Influence of externally imposed and internally generated electrical fields on grain growth, diffusional creep, sintering and related phenomena in ceramics. J Am Ceram Soc 2011;94:1941-65.

9. Naik K, Sglavo VM, Raj R. Flash sintering as a nucleation phenomenon and a model thereof J Eur Ceram Soc 2014;34:4063-4067.

10. Grasso S, Sakka Y, Rendtorff N, Hu CF, Maizza G, Borodianska H and Vasylkiv O. Modeling of the temperature distribution of flash sintered zirconia. J Ceram Soc Jap 2011; 119: 144-146.

11. Fowler AC, Frigaard I and Howison SD. Temperature surges in current-limiting circuit devices. S I A M J Appl Math 1992; 52: 998-1011.

12. Lacey AA. Thermal runaway in a non-local problem modelling Ohmic heating: Part I: Model derivation and some special cases. Eur J App Math 1995; 6:127-144. 
13. Wade WR. Measurements of total hemispherical emissivity of several stably oxidized metals and some refactory oxide coatings. NASA technical document 19980228304 (1959).

14. Ferriere A, Lestrade $L$ and Robert J-F. Optical Properties of plasma-sprayed ZrO2-Y2O3 at high temperature for solar applications. J. Solar Energy Engineering 2000; 122: 9-13.

15. See for example: http://www.omega.co.uk/literature/transactions/volume1/emissivityb.html

16. Tanaka H, Sawai S, Morimoto $\mathrm{K}$ and Hisano K. Measurement of spectral emissivity and thermal conductivity of ziconia by thermal radiation calorimetry. J Therm Anal Cal 2001; 64:867-872.

17. Koh JCY and Fortini A. Prediction of thermal conductivity and electrical resistivity of porous metallic materials. Int J Heat Mass Transfer 1973; 16: 2013-1022.

18. http://www.engineeringtoolbox.com/convective-heat-transfer-d_430.html

19. Motohashi Y, Shibata T, Baba S, Ishihara M and Hoshiya T. Thermal Conductivity of Superplastically Deformed 3Y-TZP. Materials Transactions 2002; 43: 2473-2479.

20. Francis JSC, Cologna M and Raj R. Particle size effects in flash sintering. J Eur Ceram Soc 2012; 32: 3129-3136.

21. Cologna M, Prette ALG and Raj R. Flash-sintering of cubic yttria-stabilized zirconia at $750{ }^{\circ} \mathrm{C}$ for possi use in SOFC manufacturing. J Am Ceram Soc 2011; 94: 316-319.

22. M'Peko JC, Francis SC and Raj R. Field-assisted sintering of undoped BaTiO3: microstructure evolution and dielectric permittivity. J Eur Ceram Soc 2014; 34: 3655-3660.

23. Lin TF, Hu CT, Lin IN. Influence of stoichiometry on the microstructure and positive temperature-coefficient of resistivity of semiconducting barium-titanate ceramics. J Am Ceram Soc 1990; 73: 531-36.

24. Lebrun JM and Raj R. A First report of photoemission in experiments related to flash sintering. J Am Ceram Soc 2014; 97: 2427-2430.

25. Muccillo R, Kleitz M and Muccillo ENS. Flash grain welding in yttria stabilized zirconia. J Eur Ceram Soc 2011; 31 1517-1521.

26. Yamamoto O, Takeda Y, Kanno R, Kohno K and Kamiharai T. J Mat Sci Lett 1989; 8: 198-200.

27. Baraki R, Schwarz S and Guillon O. J Am Ceram Soc 2012; 95: 75-78.

28. Akdoğan EK, Şavklıyıldız I, Biçer H, Paxton W, Toksoy F, Zhong Z and Tsakalakos T. Anomalous lattice expansion in yttria stabilized zirconia under simultaneous applied electric and thermal fields: A time-resolved in situ energy dispersive $x$-ray diffractometry study with an ultrahigh energy synchrotron probe. J App Phys 2013; 113: 233503. 